Preprint typeset in JHEP style - HYPER VERSION

\title{
Lunin-Maldacena Deformations with Three Parameters
}

\author{
Aybike Çatal-Özer \\ School of Mathematics, Trinity College Dublin \\ Dublin 2 IRELAND \\ e-mail: aybike@maths.tcd.ie
}

\begin{abstract}
We examine the solution generating symmetries by which Lunin and Maldacena have generated the gravity duals of $\beta$-deformations of certain field theories. We identify the $O(2,2, \mathbb{R})$ matrix, which acts on the background matrix $E=g+B$, where $g$ and $B$ are the metric and the B-field of the undeformed background, respectively. This simplifies the calculations and makes some features of the deformed backgrounds more transparent. We also find a new three-parameter deformation of the Sasaki-Einstein manifolds $T^{1,1}$ and $Y^{p, q}$. Following the recent literature on the three-parameter deformation of $A d S_{5} \times S^{5}$, one would expect that our new solutions should correspond to non-supersymmetric marginal deformations of the relevant dual field theories.
\end{abstract}

KEYWORDS: String duality, Gauge-gravity correspondence. 


\section{Contents}

1. Introduction 1

2. A Closer Look at The Solution Generating Symmetries 2

3. A Shortcut to the Lunin-Maldacena Backgrounds 6

$3.1 A d S_{5} \times T^{1,1}$

$3.2 \quad A d S_{5} \times Y^{p, q}$

$3.3 \quad A d S_{5} \times S^{5}$

4. Three Parameter Generalizations 9

4.1 Three-parameter deformation of $A d S_{5} \times S^{5}$ : Frolov's solution 9

4.2 Three-parameter deformation of $T^{1,1}$ and $Y^{p, q}$ : New solutions 11

5. Ramond-Ramond Fields 13

6. Regularity of Solutions 16

7. Conclusions 17

\section{Introduction}

In a recent paper, Lunin and Maldacena studied a specific type of deformation of certain field theories and presented a method to find the gravity duals of the deformations [1]. The type of deformations they consider apply to both conformal and non-conformal field theories and is such that the resulting theory preserves a global $U(1) \times U(1)$ symmetry. The effect of the deformation in the Lagrangian is to introduce phases depending on the order of fields charged under this global symmetry. Such deformations are sometimes called $\beta$ deformations, as they are characterized by a complex parameter, usually called $\beta$. In the particular case when $\beta$ is real, they are usually called $\gamma$ deformations. In this paper, we will be interested only in real deformations. The prescription Lunin and Maldacena gave works when the global $U(1) \times U(1)$ is realized geometrically on the gravity side. In this case, the geometry of the gravity background contains a 2-torus and the new gravity solutions corresponding to the deformed field theory are generated by using an $S L(2, \mathbb{R})$ symmetry associated with the 2-torus. However, this $S L(2, \mathbb{R})$ is not to be confused with the geometric $S L(2, \mathbb{R})$ acting on the complex structure modulus. It rather acts on the Kähler modulus $\rho$, whose real and imaginary parts are the value of the $B$ field on the 2 -torus and the volume of the 2-torus, respectively. This is the non-geometric part of the 
symmetry group $O(2,2, \mathbb{R})$ associated with the two commuting isometries. We will discuss this further in Section 2.

Later in [2], Frolov examined the deformed gravity solutions of [1] and showed that the classical bosonic string theory in this background exhibits a Lax pair and as such, is integrable. The Bethe ansatz for the integrable spin chain was presented in [3] and the string Bethe equations were solved explicitly. Again in [2], Frolov generalized the deformed gravity solutions of [1] by introducing two more parameters and found a 3parameter family of deformations of $A d S_{5} \times S^{5}$, which he proposed as the gravity dual of a non-supersymmetric marginal deformation of $\mathcal{N}=4 \mathrm{SYM}$, at least in the large $\mathrm{N}$ limit. The integrable spin chain corresponding to this background and the Bethe ansatz were constructed in [4]. Further checks of AdS/CFT correspondence for the new 3-parameter family of deformations were performed in [5]. For more on $\beta$-deformations and their gravity duals, see for example [6, 1, 8, 9, 10, 11, 12, 13, 14, 15, 16]. Also see the very recent 17] for more on Lunin-Maldacena deformations with several parameters.

One of our aims in this paper is to have a closer look at the underlying symmetries by which the new gravity solutions have been generated in [1]. In particular, we will find the $O(2,2, \mathbb{R})$ matrix acting on the background matrix $E=g+B$, so that we obtain the solutions in an easier way. This also makes some features of the new backgrounds more transparent. For example, the transformation of the coordinates and the momenta and winding charges, which Frolov utilized in writing the Lax pair for the deformed $A d S_{5} \times S^{5}$, follows easily from the properties of the $O(2,2, \mathbb{R})$ matrix we find.

After we identify the $O(2,2, \mathbb{R})$ matrix in question, we are immediately led to our main purpose, which is to find the three-parameter deformations of the Sasaki-Einstein manifolds $T^{1,1}$ and $Y^{p, q}$, whose associated $A d S_{5}$ geometries are the near horizon limit of a stack of $\mathrm{N}$ D-branes placed at the tip of their Calabi-Yau cones. The dual field theories were found in [18] for $T^{1,1}$ and [19, 20] for $Y^{p, q}$. One would expect, following [2, 4, 5] that the new 3-parameter deformations we find here should correspond to non-supersymmetric marginal deformations of the dual field theory.

The plan of the paper is as follows. In the next section, we will examine the solution generating symmetries used in [1] and identify the $O(2,2, \mathbb{R})$ matrix, which acts on $E=$ $g+B$. In section 3, we will reproduce the results of [1] by the action of the matrix we find. In section 4, we first discuss the 3-parameter generalization of [2]. Then we present our new solutions, which are the 3-parameter deformations of the Sasaki-Einstein manifolds $T^{1,1}$ and $Y^{p, q}$. All our discussions until section 5 focus on the NS sector of the solutions, whereas section 5 is devoted to the discussion of the RR sector. After discussing the regularity of the solutions in section 6 , we conclude with section 7 .

\section{A Closer Look at The Solution Generating Symmetries}

Perhaps the best way to study the solution generating transformations of [1] is to look at the U-duality group of the eight dimensional TypeII string theory: $S L(2, \mathbb{R}) \times S L(3, \mathbb{R})$. Obviously, we are not being rigorous by calling this solution generating group the U-duality group, as in fact the U-duality group is the discrete $S L(2, Z) \times S L(3, Z)$ [21]. However, for 
ease of language, we will refer to the above group as the U-duality group just as we will, throughout the text, refer to the solution generating $O(d, d, \mathbb{R})$ as the T-duality group. Let us first examine the subgroup $S O(2,2, \mathbb{R}) \simeq S L(2, \mathbb{R})_{\tau} \times S L(2, \mathbb{R})_{\rho}$, which is the "trivial" part of the T-duality group $O(2,2, \mathbb{R})$ acting within IIA or IIB $^{1}$. Here the subindexes $\tau$ and $\rho$ refers to the fact that the first $S L(2, \mathbb{R})$ acts on the complex structure modulus $\tau$ of the internal 2-torus, whereas the second one acts on its Kähler modulus $\rho . S L(2, \mathbb{R})_{\tau}$ is the geometric part of the T-duality associated with invariance under large diffeomorphisms of the two torus on which the ten dimensional type IIB string theory has been compactified. Its action on the complex structure modulus $\tau=\tau_{1}+i \tau_{2}$ and the background matrix $E=g+B$ is:

$$
\tau \longrightarrow \frac{a \tau+b}{c \tau+d}, \quad E \longrightarrow A E A^{t}
$$

Here

$$
A=\left(\begin{array}{ll}
a & b \\
c & d
\end{array}\right)
$$

is an $S L(2, \mathbb{R})$ matrix embedded in $O(2,2, \mathbb{R})$ as

$$
\left(\begin{array}{cc}
A & 0_{2} \\
0_{2} & \left(A^{t}\right)^{-1}
\end{array}\right),
$$

so that the background matrix $E$ transforms as in (2.1).

On the other hand, the $S L(2, \mathbb{R})_{\rho}$ acts on the Kähler modulus $\rho=\rho_{1}+i \rho_{2}=B_{12}+i \sqrt{g}$ as

$$
\rho \longrightarrow \frac{a \rho+b}{c \rho+d}
$$

with $a d-b c=1$. It is important to know how $S L(2, \mathbb{R})_{\rho}$ is embedded into $O(2,2, \mathbb{R})$. One can see that the the action of $S L(2, \mathbb{R})_{\rho}$ on the background matrix $E$ is through the following $O(2,2, \mathbb{R})$ matrix

$$
T=\left(\begin{array}{ll}
A & B \\
C & D
\end{array}\right)=\left(\begin{array}{rrrr}
a & 0 & 0 & b \\
0 & a & -b & 0 \\
0 & -c & d & 0 \\
c & 0 & 0 & d
\end{array}\right)
$$

It is easy to check that as the background matrix transforms as 22

$$
E \longrightarrow(A E+B)(C E+D)^{-1} \equiv \frac{A E+B}{C E+D}
$$

with $A, B, C, D$ as in (2.4), $\rho_{1}=B_{12}$ and $\rho_{2}=\sqrt{g}$ transform as

$$
\rho_{1} \longrightarrow \frac{a c\left(\rho_{1}^{2}+\rho_{2}^{2}\right)+(a d+b c) \rho_{1}+b d}{c^{2}\left(\rho_{1}^{2}+\rho_{2}^{2}\right)+2 d c \rho_{1}+d^{2}}
$$

\footnotetext{
${ }^{1}$ Obviously, $O(2,2, Z)$ also has matrices with determinant -1 , including the transformation which exchanges the Kähler and the complex structure moduli. Sometimes only this transformation is referred to as being the T-duality transformation (as in [2), and sometimes it is referred to as the non-trivial part of the T-duality group. For an extensive review of T-duality, see [22].
} 


$$
\rho_{2} \longrightarrow \frac{\rho_{2}}{c^{2}\left(\rho_{1}^{2}+\rho_{2}^{2}\right)+2 d c \rho_{1}+d^{2}}
$$

which is equivalent to (2.3).

Yet another $S L(2, \mathbb{R})$ symmetry of the IIB string theory is the S-duality, which already exists in ten dimensions and whose $Z_{2}$ part acts on the string coupling as a strong-weak coupling duality. This group, which we will denote by $S L(2, \mathbb{R})_{s}$ combines with the nongeometric $S L(2, \mathbb{R})_{\rho}$ to form the $S L(3, \mathbb{R})$ part of the U-duality group. From the point of view of type IIA string theory, this $S L(3, \mathbb{R})$ is the geometric part of the U-duality group, associated with the large diffeomorphisms of the three torus on which the eleven dimensional M-theory has been compactified. On the other hand, the geometric $S L(2, \mathbb{R})_{\tau}$ of IIB is mapped under T-duality to a non-geometric $S L(2, \mathbb{R})$ which acts on the Kähler modulus of Type IIA.

The new solutions of [1] are generated through the action of $S L(3, \mathbb{R})$. The action of $S L(2, \mathbb{R})_{s}$ generates new solutions which correspond to a $\beta$-deformation of the field theory with a complex parameter $\beta=\sigma$. In this paper, we will only be interested in real deformations.

The supergravity solutions of Lunin and Maldacena, which correspond to $\beta$-deformations with a real parameter $\beta=\gamma$ of the dual field theory are obtained by the action of $S L(2, \mathbb{R})_{\rho}$ on $\rho$ as in (2.3), with the particular choice $a=d=1, b=0, c=\gamma$ (so that the resulting solutions are regular). From (2.4) we see that the relevant T-duality matrix which acts on the background matrix as in (2.5) is

$$
T=\left(\begin{array}{cc}
1_{2} & 0_{2} \\
\Gamma & 1_{2}
\end{array}\right)
$$

where $1_{2}$ and $0_{2}$ are the $2 \times 2$ identity and zero matrices respectively, whereas

$$
\Gamma=\left(\begin{array}{rr}
0 & -\gamma \\
\gamma & 0
\end{array}\right) .
$$

Perhaps it is useful at this point to recall once again that this is merely a solution generating action and not a T-duality one. In fact, as was explained in [1], on the field theory side the corresponding deformation of the field theory superpotential is

$$
\operatorname{Tr}\left(\Phi_{1} \Phi_{2} \Phi_{3}-\Phi_{1} \Phi_{3} \Phi_{2}\right) \rightarrow \operatorname{Tr}\left(e^{i \pi \gamma} \Phi_{1} \Phi_{2} \Phi_{3}-e^{-i \pi \gamma} \Phi_{1} \Phi_{3} \Phi_{2}\right) .
$$

When $\gamma$ is integer, (2.6) becomes an element of the T-duality group $O(2,2, Z)$ of IIB string theory and the superpotential remains the same. There is no deformation at all. Of course, this only makes sense. From the world-sheet point of view, $O(d, d, \mathbb{R})$ acts on the moduli space of $2 \mathrm{~d} \mathrm{CFT's}^{2}$ (on the world-sheet of the string theory whose target space is the background in question), taking one CFT to another connected to it by an exactly marginal deformation [22]. On the field theory side, this corresponds to starting

\footnotetext{
${ }^{2}$ When the background is flat, with the topology of $T^{d}$, the moduli space is $O(d, d, \mathbb{R}) / O(d, \mathbb{R}) \times O(d, \mathbb{R})$. For curved backgrounds with $d$ commuting isometries, it is more complicated but there is still a local $O(d, d, \mathbb{R})$ action on the moduli space.
} 
with the four dimensional $\mathcal{N}=4$ SCFT and deforming it to an $\mathcal{N}=1$ SCFT with $\gamma$ parameterizing the deformation (for the particular example of [6]). When $\gamma$ is integer, there is no deformation and the four dimensional conformal field theory remains the same, just like the discrete $O(d, d, Z)$ maps the world-sheet CFT to a physically equivalent one.

Let us have a closer look at $T$ in (2.6). It can be factorized in terms of the generators of $O(d, d, \mathbb{R})$ in several different ways. One way is

$$
T=g_{D_{1}} \cdot S \cdot g_{D_{1}}
$$

where $S$ is as in (2.2) with

$$
A=\left(\begin{array}{ll}
1 & \gamma \\
0 & 1
\end{array}\right)
$$

and $g_{D i}$ is the matrix of the $R \rightarrow 1 / R$ transformation, called the "factorized duality" in [22], along the $i$ th direction:

$$
\left(\begin{array}{cc}
1_{2}-e_{i} & e_{i} \\
e_{i} & 1_{2}-e_{i}
\end{array}\right)
$$

$e_{i}$ being the $2 \times 2$ matrix with all entries 0 except $i$ th entry, which is 1 . So, $T$ corresponds to a factorized duality along one leg of the 2-torus, an $S L(2, \mathbb{R})$ transformation and another factorized duality along the same leg. This is the interpretation that was used in [2]. On the other hand, $T$ can also be factorized as

$$
T=\left(\begin{array}{cc}
0 & 1_{2} \\
1_{2} & 0
\end{array}\right) \cdot\left(\begin{array}{cc}
1_{2} & \Gamma \\
0 & 1_{2}
\end{array}\right) \cdot\left(\begin{array}{cc}
0 & 1_{2} \\
1_{2} & 0
\end{array}\right) .
$$

This is a volume inversing factorized duality along both legs of the torus, a geometric shift of the B-field, and then dualizing back along the two legs. This factorization will generalize to the 3-parameter deformations, as we will see in section 4 .

For future use, let us note how the momenta $p^{i}$ and the winding charges $w^{i}, i=$ $1, \cdots, d, \quad d=\operatorname{dim} T^{d}$ associated with the world-sheet currents $\partial_{t} x^{i}$ and $\partial_{s} x^{i}$ transform under the action of a T-duality matrix of the type (2.6). Let $Z$ be the $2 d$-dimensional vector

$$
Z=\left(\begin{array}{c}
w^{i} \\
p^{i}
\end{array}\right) .
$$

Under the action of a generic $T \in O(d, d, \mathbb{R})$, it transforms as 22$]$

$$
Z \longrightarrow\left(T^{t}\right)^{-1} Z
$$

In our case, in which $d=2$ and $T$ is of the form (2.6), we have

$$
\left(\begin{array}{c}
w^{1} \\
w^{2} \\
p^{1} \\
p^{2}
\end{array}\right) \longrightarrow\left(\begin{array}{c}
w^{1}-\gamma p^{2} \\
w^{2}+\gamma p^{1} \\
p^{1} \\
p^{2}
\end{array}\right)
$$




\section{A Shortcut to the Lunin-Maldacena Backgrounds}

Now that we have the T-duality matrix whose action generates the new solutions we can reproduce the Lunin-Maldacena backgrounds in an easier way. Below we will demonstrate this briefly so that our discussions for the generalized case will be more transparent. In this section, we will only be concerned with the NS sector, leaving the transformation of the RR fields to section 5. We are leaving the discussion of $A d S_{5} \times S^{5}$ to the end, as it involves an extra feature, which will lead us to our new 3-parameter deformations.

\section{1 $A d S_{5} \times T^{1,1}$}

Our first example is $A d S_{5} \times T^{1,1}$, where $T^{1,1}$ is the coset space $(S U(2) \times S U(2)) / U(1)$. This is the dual of $\mathcal{N}=1$ supersymmetric Yang-Mills theory, which arises from a stack of $\mathrm{N}$ D-branes at the tip of the conifold, the conic Calabi-Yau 3-fold whose base space is $T^{1,1}$ 18]. The metric of $A d S_{5} \times T^{1,1}$ is

$$
\frac{d s^{2}}{R^{2}}=d s_{A d S}^{2}+\frac{1}{6} \sum_{i=1}^{2}\left(d \theta_{i}^{2}+\sin ^{2} \theta_{i} d \phi_{i}^{2}\right)+\frac{1}{9}\left(d \psi+\cos \theta_{1} d \phi_{1}+\cos \theta_{2} d \phi_{2}\right)^{2} .
$$

This is clearly an $S^{1}$ bundle over $S^{2} \times S^{2}$ with $\psi$ parameterizing the fiber circle which winds over the two base spheres once ${ }^{3}$. The isometry group of $T^{1,1}$ is $S U(2) \times S U(2) \times U(1)$ and in particular, it has three commuting Killing vectors: $\partial / \partial \phi_{1}, \partial / \partial \phi_{2}, \partial / \partial \psi$. [1] used the first two of them in order to generate new solutions corresponding to exactly marginal real $\gamma$-deformations of the field theory. Now we will see how the action of (2.6) does indeed reproduce their result.

First note that as $S^{1}$ is nontrivially fibered over the two isometry directions $\phi_{1}$ and $\phi_{2}$, we cannot simply use the matrix in (2.6) but rather should embed it in $O(3,3, \mathbb{R})$. The rules of this were given in [23] and the result is

$$
T=\left(\begin{array}{cc}
1_{3} & 0_{3} \\
\Gamma & 1_{3}
\end{array}\right)
$$

with $1_{3}$ and $0_{3}$ being $3 \times 3$ identity and zero matrices and $\Gamma$ becomes:

$$
\Gamma=\left(\begin{array}{ccc}
0 & -\gamma & 0 \\
\gamma & 0 & 0 \\
0 & 0 & 0
\end{array}\right)
$$

Applying this to the background matrix ${ }^{4}$

$$
E=R^{2}\left(\begin{array}{ccc}
\frac{c_{1}^{2}}{9}+\frac{s_{1}^{2}}{6} & \frac{c_{1} c_{2}}{9} & \frac{c_{1}}{9} \\
\frac{c_{1} c_{2}}{9} & \frac{c_{2}^{2}}{9}+\frac{s_{2}^{2}}{6} & \frac{c_{2}}{9} \\
\frac{c_{1}}{9} & \frac{c_{2}}{9} & \frac{1}{9}
\end{array}\right)
$$

\footnotetext{
${ }^{3}$ When the Hopf numbers of the $S^{1}$ bundle over the base 2-spheres are $p$ and $q$ the above generalizes to the manifolds $T^{p, q}$.

${ }^{4}$ Here we use the notation of $[1]: c_{i}=\cos \theta_{i}$ and $s_{i}=\sin \theta_{i}$.
} 
we obtain the new background matrix $E^{\prime}=g^{\prime}+B^{\prime}=E .\left(\Gamma E+1_{3}\right)^{-1}$ from which we read

$$
\begin{gathered}
\frac{d s^{2}}{R^{2}}=d s_{A d S}^{2}+G\left[\frac{1}{6} \sum_{i=1}^{2}\left(G^{-1} d \theta_{i}^{2}+\sin ^{2} \theta_{i} d \phi_{i}^{2}\right)+\frac{1}{9}\left(d \psi+\cos \theta_{1} d \phi_{1}+\cos \theta_{2} d \phi_{2}\right)^{2}+\hat{\gamma}^{2} \frac{s_{1}^{2} s_{2}^{2}}{324} d \psi^{2}\right] . \\
\frac{B}{R^{2}}=\hat{\gamma} G\left[\left(\frac{s_{1}^{2} s_{2}^{2}}{36}+\frac{c_{1}^{2} s_{2}^{2}+c_{2}^{2} s_{1}^{2}}{54}\right) d \phi_{1} \wedge d \phi_{2}+\frac{s_{1}^{2} c_{2}}{54} d \phi_{1} \wedge d \psi-\frac{c_{1} s_{2}^{2}}{54} d \phi_{2} \wedge d \psi\right]
\end{gathered}
$$

Here

$$
G=\operatorname{det}\left(\Gamma E+1_{3}\right)^{-1}=\left(1+\hat{\gamma}^{2}\left(\frac{c_{1}^{2} s_{2}^{2}+c_{2}^{2} s_{1}^{2}}{54}+\frac{s_{1}^{2} s_{2}^{2}}{36}\right)\right)^{-1}, \quad \hat{\gamma}=R^{2} \gamma
$$

The dilaton always transforms as ${ }^{5}$ [22]

$$
e^{2 \Phi} \longrightarrow \frac{e^{2 \Phi}}{\operatorname{det}\left(\Gamma E+1_{3}\right)}
$$

so we have $e^{2 \Phi^{\prime}}=G e^{2 \Phi}$. This is the exact same background as in [1], although presented in a different way ${ }^{6}$.

\section{$3.2 A d S_{5} \times Y^{p, q}$}

The next backgrounds we will consider are the recently discovered Sasaki-Einstein manifolds $Y^{p, q}$ 24, 19. The dual field theory arises from a stack of $\mathrm{N}$ D-branes placed at the tip of the toric Calabi-Yau cone over $Y^{p, q}[19,20]$. The metric is

$$
\begin{aligned}
\frac{d s^{2}}{R^{2}}= & d s_{A d S_{5}}^{2}+\frac{1-y}{6}\left(d \theta^{2}+s_{\theta}^{2} d \phi^{2}\right)+\frac{1}{w(y) q(y)} d y^{2}+\frac{q(y)}{9}\left(d \psi-c_{\theta} d \phi\right)^{2} \\
& +w(y)\left[l d \alpha+f(y)\left(d \psi-c_{\theta} d \phi\right)\right]^{2}
\end{aligned}
$$

Here

$$
\begin{gathered}
w(y)=\frac{2\left(a-y^{2}\right)}{1-y}, \quad q(y)=\frac{a-3 y^{2}+2 y^{3}}{a-y^{2}}, \quad f(y)=\frac{a-2 y+y^{2}}{6\left(a-y^{2}\right)} \\
l=\frac{q}{3 q^{2}-2 p^{2}+p\left(4 p^{2}-3 q^{2}\right)^{1 / 2}} .
\end{gathered}
$$

So the background matrix $E=g+b=g$ is

$$
E=R^{2}\left(\begin{array}{lll}
g_{11} & g_{12} & g_{13} \\
g_{12} & g_{22} & g_{23} \\
g_{13} & g_{23} & g_{33}
\end{array}\right),
$$

where

$$
\begin{aligned}
& g_{11}=l^{2} w(y), \quad g_{22}=\frac{1-y}{6} s_{\theta}^{2}+\frac{q(y)}{9} c_{\theta}^{2}+w(y) f(y)^{2} c_{\theta}^{2}, \quad g_{12}=-l w(y) f(y) c_{\theta} \\
& g_{33}=\frac{q(y)}{9}+w(y) f(y)^{2}, \quad g_{13}=l w(y) f(y), \quad g_{23}=-\frac{q(y)}{9} c_{\theta}-w(y) f(y)^{2} c_{\theta} .
\end{aligned}
$$

\footnotetext{
${ }^{5}$ We want the asymptotic value $\Phi_{0}$ of the dilaton to have the same value as in the $A d S_{5} \times X_{5}$ case, as the exactly marginal operator by which the field theory is deformed does not couple to the dilaton. As a result, $R=\left(4 \pi e^{\Phi_{0}} N\right)^{1 / 4}$ of the deformed background is the same as that of the original background.

${ }^{6}$ Except for an overall $G^{-1 / 4}=e^{-\Phi^{\prime} / 2} / e^{-\Phi / 2}$ factor, as we are presenting our results in the string frame unlike in [1], where it is presented in the Einstein frame.
} 
We find the new background metric and the B-field from the symmetric and the antisymmetric parts of $E^{\prime}=E\left(\Gamma E+1_{3}\right)^{-1}$, where $\Gamma$ is as in (3.3). First let us write the determinant of $G=\operatorname{det}\left(\Gamma E+1_{3}\right)^{-1}$.

$$
G=\frac{1}{1+\hat{\gamma}^{2} \Delta}
$$

where

$$
\Delta=\frac{2 q(y) c_{\theta}^{2}+3(1-y) s_{\theta}^{2}}{9(1-y)}\left(a-y^{2}\right) l^{2}, \quad \hat{\gamma}=R^{2} \gamma
$$

As mentioned in the previous subsection the dilaton will simply transform as $e^{2 \Phi} \rightarrow G e^{2 \Phi}$. Doing the matrix multiplication we also find

$$
\begin{aligned}
g_{i j} & \longrightarrow G g_{i j}, \quad \text { except } g_{33} \\
g_{33} & \longrightarrow G\left(g_{33}+\hat{\gamma}^{2} e\right)
\end{aligned}
$$

where $e$ is the determinant of $E$ in 3.10

$$
\begin{aligned}
e=\frac{\operatorname{det} E}{R^{6}} & =g_{33}\left(g_{11} g_{22}-g_{12}^{2}\right)+2 g_{12} g_{13} g_{23}-g_{13}^{2} g_{22}-g_{23}^{2} g_{11} \\
& =\frac{1}{27} l^{2}\left(2 y^{3}-3 y^{2}+a\right) s_{\theta}^{2}
\end{aligned}
$$

On the other hand we find

$$
\frac{B}{R^{2}}=B_{12} d \alpha \wedge d \phi+B_{13} d \alpha \wedge d \psi+B_{23} d \phi \wedge d \psi
$$

where

$$
\begin{aligned}
& B_{12}=\hat{\gamma}\left(g_{11} g_{22}-\left(g_{12}\right)^{2}\right)=\hat{\gamma} \Delta, \\
& B_{13}=\hat{\gamma}\left(g_{11} g_{23}-g_{12} g_{13}\right)=\hat{\gamma} \frac{2 l^{2}\left(a+2 y^{3}-3 y^{2}\right) c_{\theta}}{9(y-1)} \\
& B_{23}=\hat{\gamma}\left(g_{12} g_{23}-g_{22} g_{13}\right)=-\hat{\gamma} \frac{l\left(a-2 y+y^{2}\right) s_{\theta}^{2}}{18} .
\end{aligned}
$$

One can check that the new background is exactly the same as that presented in [1].

\section{$3.3 A d S_{5} \times S^{5}$}

Finally we consider the background $A d S_{5} \times S^{5}$ whose metric is

$$
\frac{d s^{2}}{R^{2}}=d s_{A d S_{5}}^{2}+\sum_{i=1}^{3} d \mu_{i}^{2}+\mu_{i}^{2} d \phi_{i}^{2}, \quad \text { with } \quad \sum_{i} \mu_{i}^{2}=1 .
$$

When we perform the T-duality transformation with the matrix (3.2) we see that the resulting background is not the same as in [1]. After a moment's thought, one realizes that the T-duality matrix, which generates the deformed solution in [1] is in fact

$$
T=\left(\begin{array}{cc}
1_{3} & 0_{3} \\
\Gamma & 1_{3}
\end{array}\right)
$$


where

$$
\Gamma=\left(\begin{array}{ccc}
0 & -\gamma & \gamma \\
\gamma & 0 & -\gamma \\
-\gamma & \gamma & 0
\end{array}\right)
$$

Of course, first doing a coordinate transformation, then a T-duality of the form (3.2) and an inverse coordinate transformation to the original coordinates will give a T-duality transformation whose matrix is

$$
\left(\begin{array}{cc}
1_{3} & 0_{3} \\
A^{t} \Gamma A & 1_{3}
\end{array}\right) .
$$

So, with an appropriate choice of coordinate transformations, one can obtain a T-duality matrix of the form (3.18), starting from (3.2). This is what was done in [1], see for example [2].

Applying (3.18) one obtains the background in [1]:

$$
\begin{aligned}
d s^{2} & =R^{2}\left[d s_{A d S_{5}}^{2}+\sum_{i}\left(d \mu_{i}^{2}+G \mu_{i}^{2} d \phi_{i}^{2}\right)+\hat{\gamma}^{2} G \mu_{1}^{2} \mu_{2}^{2} \mu_{3}^{2}\left(\sum_{i} d \phi_{i}\right)^{2}\right] \\
B & =\hat{\gamma} R^{2} G\left(\mu_{1}^{2} \mu_{2}^{2} d \phi_{1} \wedge d \phi_{2}+\mu_{2}^{2} \mu_{3}^{2} d \phi_{2} \wedge d \phi_{3}+\mu_{3}^{2} \mu_{1}^{2} d \phi_{3} \wedge d \phi_{1}\right) \\
e^{2 \Phi^{\prime}} & =G e^{2 \Phi} \\
G & =\left(1+\hat{\gamma}^{2}\left(\mu_{1}^{2} \mu_{2}^{2}+\mu_{2}^{2} \mu_{3}^{3}+\mu_{1}^{2} \mu_{3}^{2}\right)\right)^{-1}, \quad \hat{\gamma}=R^{2} \gamma
\end{aligned}
$$

At this point, one can readily guess how the generalizations with three parameters will arise.

\section{Three Parameter Generalizations}

\subsection{Three-parameter deformation of $A d S_{5} \times S^{5}$ : Frolov's solution}

As we discussed above, deformations of the $A d S_{5} \times S^{5}$ background can be obtained by transforming with the T-duality matrix (3.18), which is also equivalent to performing a coordinate transformation, a factorized duality along one isometry direction, a geometric shift, then dualizing back along the same isometry direction and finally the inverse coordinate transformation. Frolov applied the latter on all the 2-tori embedded in the 3-torus and found a 3-parameter family of deformations of the $A d S_{5} \times S^{5}$ background [2]. As one might guess from our previous discussions, it is equivalent to generating a new solution by the action of the T-duality matrix

$$
\left(\begin{array}{cc}
1_{3} & 0_{3} \\
\Gamma & 1_{3}
\end{array}\right)
$$

where $\Gamma$ is now ${ }^{7}$

$$
\left(\begin{array}{ccc}
0 & -\gamma_{3} & \gamma_{2} \\
\gamma_{3} & 0 & -\gamma_{1} \\
-\gamma_{2} & \gamma_{1} & 0
\end{array}\right)
$$

\footnotetext{
${ }^{7}$ Very recently, $d(d-1) / 2$ parameter deformations of a background with $d$ commuting $U(1)$ isometries was studied in 17]. We would expect that such deformed solutions should be generated by the action of an $O(d, d, \mathbb{R})$ matrix of the same form, where $\Gamma$ is now a $d$-dimensional antisymmetric matrix with $d(d-1) / 2$ independent parameters.
} 
Applying the T-duality matrix (4.1) to the background matrix of (3.17) we obtain exactly the solution of Frolov, whose NS sector is:

$$
\begin{aligned}
d s^{2} & =R^{2}\left[d s_{A d S_{5}}^{2}+\sum_{i}\left(d \mu_{i}^{2}+G \mu_{i}^{2} d \phi_{i}^{2}\right)+G \mu_{1}^{2} \mu_{2}^{2} \mu_{3}^{2}\left(\sum_{i} \hat{\gamma}_{i} d \phi_{i}\right)^{2}\right] \\
B & =R^{2} G\left(\hat{\gamma}_{3} \mu_{1}^{2} \mu_{2}^{2} d \phi_{1} \wedge d \phi_{2}+\hat{\gamma}_{1} \mu_{2}^{2} \mu_{3}^{2} d \phi_{2} \wedge d \phi_{3}+\hat{\gamma}_{2} \mu_{3}^{2} \mu_{1}^{2} d \phi_{3} \wedge d \phi_{1}\right) \\
e^{2 \Phi^{\prime}} & =G e^{2 \Phi} \\
G & =\left(1+\left(\hat{\gamma}_{3}^{2} \mu_{1}^{2} \mu_{2}^{2}+\hat{\gamma}_{1} \mu_{2}^{2} \mu_{3}^{3}+\hat{\gamma}_{2} \mu_{1}^{2} \mu_{3}^{2}\right)\right)^{-1}, \quad \hat{\gamma}_{i}=R^{2} \gamma_{i} .
\end{aligned}
$$

Before we move on to obtain the 3-parameter deformations of the toric backgrounds with the same method, let us pause for a moment and look at the solution generating matrix (4.1) more carefully. First of all, as the $\gamma_{i}$ are now independent, the only possible factorization of (4.1) in terms of the generators of $O(d, d, \mathbb{R})$ is

$$
\left(\begin{array}{cc}
1_{3} & 0_{3} \\
\Gamma & 1_{3}
\end{array}\right)=\left(\begin{array}{cc}
0 & 1_{3} \\
1_{3} & 0
\end{array}\right) \cdot\left(\begin{array}{cc}
1_{3} & \Gamma \\
0 & 1_{3}
\end{array}\right) \cdot\left(\begin{array}{cc}
0 & 1_{3} \\
1_{3} & 0
\end{array}\right) .
$$

So, the action of (4.1) is equivalent to a factorized duality along all the isometry directions, then doing a $\Theta$ shift $^{8}$ and finally an inverse factorized duality along all the isometry directions. So, perhaps it is convenient, in the spirit of [2], to call this a $\mathrm{T} \Theta \mathrm{T}$ transformation.

We also would like to discuss the transformation of the vector of momentum and winding charges, as this played a crucial role in [2]. Using (2.11) we see that the momenta are conserved and if $w_{i}$ are the winding numbers of the undeformed background then the deformed background has

$$
\begin{aligned}
& \phi_{1}(2 \pi)-\phi_{1}(0)=2 \pi\left(w_{1}+\gamma_{2} J_{3}-\gamma_{3} J_{2}\right) \\
& \phi_{2}(2 \pi)-\phi_{2}(0)=2 \pi\left(w_{2}+\gamma_{3} J_{1}-\gamma_{1} J_{3}\right) \\
& \phi_{3}(2 \pi)-\phi_{3}(0)=2 \pi\left(w_{3}+\gamma_{1} J_{2}-\gamma_{2} J_{1}\right) .
\end{aligned}
$$

Here $\left(J_{1}, J_{2}, J_{3}\right)$ are the conserved angular momenta ${ }^{9}$. This is the generalization of what was used in [2] in order to write down the Lax pair of the (1-parameter) deformation of the $A d S_{5} \times S^{5}$ background. Another crucial information that 2] needed to write the Lax pair was the transformation of coordinates. Under a general T-duality transformation (2.5), the metric always transforms as

$$
g \longrightarrow K^{t} g K
$$

where

$$
K=(C E+D)^{-1}
$$

Instead of this "active transformation" one can also consider the "passive transformation" of the coordinates

$$
d \phi_{i} \longrightarrow d \tilde{\phi}_{i}=K d \phi_{i}
$$

\footnotetext{
${ }^{8}$ It is standard to call the generators of $O(d, d, \mathbb{R})$ of this type $\Theta$ shifts 22.

${ }^{9}$ Here we use the letter $J$, not $p$, following [2], where $p$ is used to name the world-sheet current.
} 
so that the line element $d \phi^{t} g d \phi$ still transforms in the same way. (4.9) gives the following on-shell relations between the two coordinate systems

$$
\begin{aligned}
& d \tilde{\phi}_{1}=G\left[\left(1+\hat{\gamma}_{1}^{2} \mu_{2}^{2} \mu_{3}^{2}\right) d \phi_{1}+\left(\hat{\gamma}_{3} \mu_{2}^{2}+\hat{\gamma}_{1} \hat{\gamma}_{2} \mu_{2}^{2} \mu_{3}^{2}\right) d \phi_{2}-\left(\hat{\gamma}_{2} \mu_{3}^{2}-\hat{\gamma}_{1} \hat{\gamma}_{3} \mu_{2}^{2} \mu_{3}^{2}\right) d \phi_{3}\right] \\
& d \tilde{\phi}_{2}=G\left[-\left(\hat{\gamma}_{3} \mu_{1}^{2}-\hat{\gamma}_{1} \hat{\gamma}_{2} \mu_{1}^{2} \mu_{3}^{2}\right) d \phi_{1}+\left(1+\hat{\gamma}_{2}^{2} \mu_{1}^{2} \mu_{3}^{2}\right) d \phi_{2}+\left(\hat{\gamma}_{1} \mu_{3}^{2}+\hat{\gamma}_{2} \hat{\gamma}_{3} \mu_{1}^{2} \mu_{3}^{2}\right) d \phi_{3}\right] \\
& d \tilde{\phi}_{3}=G\left[\left(\hat{\gamma}_{2} \mu_{1}^{2}+\hat{\gamma}_{1} \hat{\gamma}_{3} \mu_{1}^{2} \mu_{2}^{2}\right) d \phi_{1}-\left(\hat{\gamma}_{1} \mu_{2}^{2}-\hat{\gamma}_{2} \hat{\gamma}_{3} \mu_{1}^{2} \mu_{2}^{2}\right) d \phi_{2}+\left(1+\hat{\gamma}_{3}^{2} \mu_{1}^{2} \mu_{2}^{2}\right) d \phi_{3}\right]
\end{aligned}
$$

where $\hat{\gamma}_{i}$ and $G$ are as in (4.3). This played a crucial role in [2] in writing the Lax pair for the strings in the deformed background.

\subsection{Three-parameter deformation of $T^{1,1}$ and $Y^{p, q}$ : New solutions}

We are finally in a position to write our new three-parameter deformations of the SasakiEinstein manifolds $T^{1,1}$ and $Y^{p, q}$. All we have to do is to generate new solutions by the action of the matrix (4.1). Following [2], one would expect our new solutions to correspond to non-supersymmetric marginal deformations of the dual field theory ${ }^{10}$. For the 3-parameter deformation of $A d S_{5} \times S^{5}$, the corresponding integrable spin chain and the Bethe ansatz were constructed in 顿. Further checks of the AdS/CFT correspondence were performed in [5]. This motivates us to study the three-parameter deformations of the toric backgrounds, even though the resulting theory will be non-supersymmetric. Note that, as the form of the solution generating matrix is the same, (4.5) and (4.9) will also remain the same. Obviously, the matrix $K$ depends on the background matrix $E$, therefore it will be more complicated in our case. Before we move on to the next point, it would be relevant to make a remark about the supersymmetry, or the lack thereof. Let's have a look back at Frolov's solution. We do not expect this solution to be supersymmetric because we have T-dualized along a $U(1)$ direction with respect to which the Killing spinors have non-vanishing Lie derivative. However, a curious thing happens when all $\gamma_{i}$ are integers. As was discussed before, in this case there is no deformation to the field theory, so the dual field theory is supersymmetric. Perhaps, this is not so curious, when one remembers the existence of supersymmetric string vacua for which the corresponding supergravity solution does not have any Killing spinors. This is the phenomena of "supersymmetry without supersymmetry", which was discussed in [26, 27]. After this remark, we simply present the new backgrounds below.

$$
\begin{aligned}
\frac{A d S_{5} \times T^{1,1}}{R^{2}}= & d s_{A d S_{5}}^{2}+G\left[\frac{1}{6} \sum_{i=1}^{2}\left(G^{-1} d \theta_{i}^{2}+s_{i}^{2} d \phi_{i}^{2}\right)+\frac{1}{9}\left(d \psi+c_{1} d \phi_{1}+c_{2} d \phi_{2}\right)^{2}+\right. \\
& \left.+\frac{s_{1}^{2} s_{2}^{2}}{324}\left(\hat{\gamma}_{3} d \psi+\hat{\gamma}_{1} d \phi_{1}+\hat{\gamma}_{2} d \phi_{2}\right)^{2}\right] \\
\frac{B}{R^{2}}= & G\left[\left(\hat{\gamma}_{3}\left(\frac{c_{1}^{2} s_{2}^{2}+c_{2}^{2} s_{1}^{2}}{54}+\frac{s_{1}^{2} s_{2}^{2}}{36}\right)-\hat{\gamma}_{2} \frac{c_{2} s_{1}^{2}}{54}-\hat{\gamma}_{1} \frac{c_{1} s_{2}^{2}}{54}\right) d \phi_{1} \wedge d \phi_{2}\right.
\end{aligned}
$$

\footnotetext{
${ }^{10}$ In $[25]$, it was shown that the spectrum of string theory in the deformed (with three parameters) flat space contains tachyons. It was argued in [17] that this does not necessarily imply the unstability of the string theory on the deformed $A d S_{5} \times S^{5}$.
} 


$$
\begin{aligned}
& +\frac{\hat{\gamma}_{3} s_{1}^{2} c_{2}-\hat{\gamma}_{2} s_{1}^{2}}{54} d \phi_{1} \wedge d \psi-\frac{\hat{\gamma}_{3} c_{1} s_{2}^{2}-\hat{\gamma}_{1} s_{2}^{2}}{54} d \phi_{2} \wedge d \psi \\
e^{2 \Phi^{\prime}}= & G e^{2 \Phi}, \quad \hat{\gamma}_{i}=R^{2} \gamma_{i} \\
G= & \left(1+\left(\hat{\gamma}_{3}^{2}\left(\frac{c_{1}^{2} s_{2}^{2}+c_{2}^{2} s_{1}^{2}}{54}+\frac{s_{1}^{2} s_{2}^{2}}{36}\right)+\hat{\gamma}_{2}^{2} \frac{s_{1}^{2}}{54}+\hat{\gamma}_{1}^{2} \frac{s_{2}^{2}}{54}-\hat{\gamma}_{3} \hat{\gamma}_{2} \frac{s_{1}^{2} c_{2}}{27}-\hat{\gamma}_{1} \hat{\gamma}_{3} \frac{c_{1} s_{2}^{2}}{27}\right)\right)^{-1}
\end{aligned}
$$

This solution reduces to that of [1] when $\gamma_{3}=\gamma$ and $\gamma_{1}=\gamma_{2}=0$.

\section{$A d S_{5} \times Y^{p, q}:$}

The background obtained by applying the solution generating matrix (4.1) has the metric components

$$
g_{i j} \longrightarrow G\left(g_{i j}+\hat{\gamma}_{i} \hat{\gamma}_{j} e\right)
$$

where $e$ is as in (3.15) and

$$
G^{-1}=1+\sum_{i} \hat{\gamma}_{i}^{2} \Delta_{i}+2 \hat{\gamma}_{1} \hat{\gamma}_{2} K_{3}+2 \hat{\gamma}_{1} \hat{\gamma}_{3} K_{2}+2 \hat{\gamma}_{2} \hat{\gamma}_{3} K_{1}
$$

with

$$
\begin{aligned}
& K_{1}=g_{12} g_{13}-g_{11} g_{23}, \quad K_{2}=g_{12} g_{23}-g_{22} g_{13}, \quad K_{3}=g_{13} g_{23}-g_{12} g_{33}, \\
& \Delta_{1}=g_{22} g_{33}-\left(g_{23}\right)^{2}, \quad \Delta_{2}=g_{11} g_{33}-\left(g_{13}\right)^{2}, \quad \Delta_{3}=g_{11} g_{22}-\left(g_{12}\right)^{2} \text {. }
\end{aligned}
$$

$\Delta_{3}=\Delta$ in 3.12 and $K_{1}, K_{2}$ were given in (3.16). On the other hand, $K_{3}=0$ and

$$
\begin{aligned}
& \Delta_{1}=\frac{(1-y) s_{\theta}^{2}}{6}\left(\frac{q(y)}{9}+w(y) f(y)^{2}\right)=\frac{\left(2+a-6 y+3 y^{2}\right) s_{\theta}^{2}}{108}, \\
& \Delta_{2}=\frac{l^{2} w(y) q(y)}{9}=\frac{2 l^{2}\left(a-3 y^{2}+2 y^{3}\right)}{9(1-y)} .
\end{aligned}
$$

The metric, B-field and the dilaton of the new solution are

$$
\begin{aligned}
\frac{d s^{2}}{R^{2}}= & G\left[d s_{A d S_{5}}^{2}+\frac{1-y}{6}\left(d \theta^{2}+s_{\theta}^{2} d \phi^{2}\right)+\frac{1}{w(y) q(y)} d y^{2}+\frac{q(y)}{9}\left(d \psi-c_{\theta} d \phi\right)^{2}\right. \\
& \left.+w(y)\left[l d \alpha+f(y)\left(d \psi-c_{\theta} d \phi\right)\right]^{2}+\frac{l^{2}\left(2 y^{3}-3 y^{2}+a\right) s_{\theta}^{2}}{27}\left(\hat{\gamma}_{1} d \alpha+\hat{\gamma}_{2} d \phi+\hat{\gamma}_{3} d \psi\right)^{2}\right] \\
\frac{B}{R^{2}}= & G\left(B_{12} d \alpha \wedge d \phi+B_{13} d \alpha \wedge d \psi+B_{23} d \phi \wedge d \psi\right) \\
e^{2 \Phi^{\prime}}= & G e^{2 \Phi}, \quad \hat{\gamma}_{i}=R^{2} \gamma_{i}
\end{aligned}
$$

where

$$
\begin{aligned}
& B_{12}=\hat{\gamma}_{3} \Delta_{3}+\hat{\gamma}_{1} K_{2}+\hat{\gamma}_{2} K_{1}, \\
& B_{31}=\hat{\gamma}_{2} \Delta_{2}+\hat{\gamma}_{1} K_{3}+\hat{\gamma}_{3} K_{1}, \\
& B_{23}=\hat{\gamma}_{1} \Delta_{1}+\hat{\gamma}_{3} K_{2}+\hat{\gamma}_{2} K_{3} .
\end{aligned}
$$

As before, this solution reduces to that of [1] when $\gamma_{3}=\gamma$ and $\gamma_{1}=\gamma_{2}=0$. 


\section{Ramond-Ramond Fields}

So far, we have only considered the NS sector of the new solutions. In section 3 , we showed that the action of the solution generating matrix (3.2) and (3.18) generates the NS sector of the Lunin-Maldacena backgrounds, which immediately ensures that the RR sectors also agree. Then in section 4, we obtained new 3 -parameter deformations of the toric backgrounds. The aim of the present section is to study the RR sector of these new solutions.

Given a T-duality matrix, which acts on the NS sector as in (2.5) one can also work out the transformation of the RR fields under the action of this matrix. This was given in [28, 29, 30, 31]. Here we will follow [31], which focuses on the T-duality transformations acting within Type IIA or Type IIB, namely those in $S O(d, d)$ with determinant 1 . Surely, our matrix of interest, (4.1) is of this type.

As was already realized in [21, 32] RR fields combine with the NS 2-form field in an appropriate way to transform under the chiral spinor representation of $O(d, d)$. The details were worked out in [31]. Here we give a brief review of their results, without presenting the details.

The first step is to combine the RR potentials $C_{p}$ of the Type II string theory (even forms in IIB and odd forms in IIA) with the NS 2-form field $B_{2}$ in the following way:

$$
\begin{array}{ll}
D_{0} & \equiv C_{0}, \quad D_{1} \equiv C_{1}, \\
D_{2} & \equiv C_{2}+B_{2} \wedge D_{0}, \quad D_{3} \equiv C_{3}+B_{2} \wedge C_{1}, \\
D_{4} & \equiv C_{4}+\frac{1}{2} B_{2} \wedge C_{2}+\frac{1}{2} B_{2} \wedge B_{2} \wedge C_{0} .
\end{array}
$$

Now introduce

$$
D \equiv \sum_{p=0}^{8} D_{p}, \quad F \equiv e^{-B_{2}} \sum_{p=0}^{8} d D_{p}=\sum_{p=0}^{8} F_{p+1} .
$$

The indices run from 0 to 8 , as we have also included the electromagnetic duals of the gauge potentials $D_{p}{ }^{11}$.

Next we introduce $2 d$ fermionic operators $\psi_{i}$ and $\psi^{i \dagger}$ satisfying

$$
\left\{\psi_{i}, \psi^{j \dagger}\right\}=\delta_{i}{ }^{j} \mathbf{1}, \quad\left\{\psi_{i}, \psi_{j}\right\}=\left\{\psi^{i \dagger}, \psi^{j \dagger}\right\}=0, \quad(i, j=1, \cdots, d)
$$

with

$$
\left(\psi_{i}\right)^{\dagger}=\psi^{i \dagger} \text {. }
$$

We construct a $2^{d}$ dimensional Fock space spanned by

$$
\left|\alpha>=\psi^{i_{1} \dagger} \cdots \psi^{i_{n} \dagger}\right| 0>\quad(n=0, \cdots, d)
$$

where we have introduced the vacuum $\mid 0>$ such that $\psi_{i}|0\rangle=0$ and $<0|0\rangle=1$. $\alpha$ in (5.4) is a multi-index $\alpha=\left(i_{1}, \cdots, i_{n}\right)$ with $\left(i_{1}<\cdots<i_{n}\right)$. Now we would like to construct

\footnotetext{
${ }^{11}$ The electromagnetic duals $D_{8-p}$ of $D_{p}$ are the potential fields obtained by solving the field equations for the latter. This ensures that $F$ defined as above satisfies $F_{10-p}=(-1)^{\left[\frac{p-1}{2}\right]} * F_{p}$ where $\left[\frac{p-1}{2}\right]$ is the first integer greater than or equal to $\frac{p-1}{2}$ [33].
} 
states in this Fock space corresponding to the form fields $D$ and $F$ in (5.2). To this end, [31] utilized the following one-to-one correspondence between the set of differential forms and the space of creation operators $\psi^{i \dagger}$ under which a differential form $\Omega$

$$
\Omega=\sum_{n} \frac{1}{n !} \Omega_{i_{1} \cdots i_{n}} d y^{i_{1}} \wedge \cdots \wedge d y^{i_{n}}=\sum_{q} \sum_{n} \frac{1}{n !} \Omega_{i_{1} \cdots i_{n}}^{(q)} d y^{i_{1}} \wedge \cdots \wedge d y^{i_{n}}
$$

is mapped to the following operator

$$
\boldsymbol{\Omega} \equiv \sum_{n} \frac{1}{n !} \Omega_{i_{1} \cdots i_{n}} \psi^{i_{1} \dagger} \cdots \psi^{i_{n} \dagger}=\sum_{q} \sum_{n} \frac{1}{n !} \Omega_{i_{1} \cdots i_{n}}^{(q)} \psi^{i_{1} \dagger} \cdots \psi^{i_{n} \dagger} .
$$

On the right hand side, the index $(q)$ indicates that the degree of $\Omega$ is $q$ as a differential form on the non-compact space, whereas $i_{1}, \cdots, i_{n}$ are compact indices. This actually gives an isomorphism as an algebra. Now, to each differential form $\Omega$ one can construct the following state

$$
|\Omega>\equiv \Omega| 0>\text {. }
$$

The main result of [31] is that the states corresponding to $D$ and $F$ in (5.2) transform under $S O(d, d)$ as

$$
|F>\longrightarrow \Lambda| F>, \quad|D>\longrightarrow \Lambda| D>
$$

where

$$
\Lambda\left|\beta>=\sum_{\alpha}\right| \alpha>S_{\alpha \beta}(\Lambda)
$$

and $S(\Lambda)=\left(S_{\alpha \beta}(\Lambda)\right)$ is the spinor representation. Here $\Lambda$ is a given $S O(d, d)$ matrix. [31] gave the explicit construction of the operators $\boldsymbol{\Lambda}$ corresponding to the generators of $S O(d, d)$.

Now, let us turn to our case of interest where $d=3$ and the $S O(3,3)$ matrix of concern is either of the form (3.2), (3.18) or (4.1). Also note that there is no B-field in the original background so the RR fields $D_{m}$ defined in (5.1) are simply equal to $C_{m}$. The operator corresponding to our solution generating matrix, which acts on the Fock space, was constructed in [31] as

$$
\boldsymbol{\Lambda}=\exp \left(\frac{1}{2} \Gamma_{m n} \psi_{m} \psi_{n}\right)
$$

From the discussion given above one can see easily that the corresponding operator acting on the differential forms is

$$
\mathbf{T}=\exp \left(\frac{1}{2} \Gamma_{m n} i_{m} i_{n}\right)
$$

where $i_{m}$ is contraction with the isometry direction $\partial / \partial y^{m}$.

In all the $A d S_{5} \times X_{5}$ backgrounds that are of interest to us here, the only RR field is $C_{4}$ with field strength

$$
F_{5}=\frac{(16 \pi N) \pi^{3}}{\operatorname{Vol}\left(X_{5}\right)}\left(w_{A d S_{5}}+* w_{A d S_{5}}\right)
$$

where $w_{A d S_{5}}$ is the volume form on $A d S_{5}$ and $*$ is the Hodge dual, so that $* w_{A d S_{5}}$ is the volume form on $X_{5}$. For example when $X_{5}=Y^{p, q}$ we have

$$
* w_{A d S_{5}}=\frac{l}{18}(1-y) s_{\theta} d \theta \wedge d y \wedge d \psi \wedge d \alpha \wedge d \phi .
$$


So, the RR fields of the new background obtained by the solution generating matrix (2.6) is

$\frac{16 \pi N}{V}\left(1-\gamma i_{\alpha} i_{\phi}\right)\left(w_{A d S_{5}}+* w_{A d S_{5}}\right)=\frac{16 \pi N}{V}\left(\left(w_{A d S_{5}}+* w_{A d S_{5}}\right)+\frac{\gamma l}{18}(1-y) s_{\theta} d \theta \wedge d y \wedge d \psi\right)$.

Here we have used

$$
V^{-1}=\frac{\operatorname{Vol}\left(S^{5}\right)}{\operatorname{Vol}\left(Y^{p, q}\right)}=\frac{\pi^{3}}{\operatorname{Vol}\left(Y^{p, q}\right)}=\left[\frac{q^{2}\left[2 p+\left(4 p^{2}-3 q^{2}\right)^{1 / 2}\right]}{3 p^{2}\left[3 q^{2}-2 p^{2}+p\left(4 p^{2}-3 q^{2}\right)^{1 / 2}\right]}\right]^{-1} .
$$

In a suitable gauge we obtain

$$
\begin{aligned}
F_{5} & =\frac{16 \pi N}{V}\left(w_{A d S_{5}}+* w_{A d S_{5}}\right) \\
C_{2} & =-\frac{8 \pi N \gamma l}{9 V}(1-y) c_{\theta} d y \wedge d \psi
\end{aligned}
$$

This is the RR fields of the one-parameter deformed background of $\| 1]^{12}$. The generalization to our case is now obvious and we obtain

$$
\begin{aligned}
& F_{5}=\frac{16 \pi N}{V}\left(w_{A d S_{5}}+* w_{A d S_{5}}\right) \\
& C_{2}=-\frac{8 \pi N l}{9 V}(1-y) c_{\theta}\left(\gamma_{3} d y \wedge d \psi+\gamma_{2} d y \wedge d \phi+\gamma_{1} d y \wedge d \alpha\right) .
\end{aligned}
$$

On the other hand, for the $T^{1,1}$ case the volume form and the total volume are

$$
* w_{A d S_{5}}=\frac{1}{108} s_{\theta_{1}} s_{\theta_{2}} d \theta_{1} \wedge d \theta_{2} \wedge d \phi_{1} \wedge d \phi_{2} \wedge d \psi \quad \text { and } \quad \operatorname{Vol}\left(T^{1,1}\right)=\frac{16 \pi^{3}}{27}
$$

The results of [1] is obtained from

$$
27 \pi N\left(1-\gamma i_{\phi_{1}} i_{\phi_{2}}\right)\left(w_{A d S_{5}}+* w_{A d S_{5}}\right)=27 \pi N\left(\left(w_{A d S_{5}}+* w_{A d S_{5}}\right)+\gamma \frac{s_{1} s_{2}}{108} d \theta_{1} \wedge d \theta_{2} \wedge d \psi\right)
$$

so that in a suitable gauge one has

$$
F_{5}=27 \pi N\left(w_{A d S_{5}}+* w_{A d S_{5}}\right), \quad C_{2}=-\frac{\gamma \pi N}{4} c_{1} s_{2} d \theta_{2} \wedge d \psi
$$

The generalizaton to the 3-parameter case is now obvious and here we present the result as

$$
\begin{aligned}
& F_{5}=27 \pi N\left(w_{A d S_{5}}+* w_{A d S_{5}}\right) \\
& C_{2}=-\frac{\pi N}{4} c_{1} s_{2}\left(\gamma_{3} d \theta_{2} \wedge d \psi+\gamma_{2} d \theta_{2} \wedge d \phi_{2}+\gamma_{1} d \theta_{2} \wedge d \phi_{1}\right)
\end{aligned}
$$

\footnotetext{
${ }^{12}$ Note that there is a sign flip for $C_{2}$. As has already been noted in [2] the sign conventions of [1] are different from the literature.
} 


\section{Regularity of Solutions}

In this section, we analyze the regularity of the new solutions. As was already mentioned in the text, under a T-duality transformation of the form (2.5), the metric always transforms as

$$
g \longrightarrow g^{\prime}=\frac{1}{(C E+D)^{t}} g \frac{1}{(C E+D)}
$$

so that the transformation of the volume form is

$$
* \mathbf{1}=\sqrt{\operatorname{det} g} d y_{1} \wedge d y_{2} \wedge d y_{3} \longrightarrow \sqrt{\operatorname{det} g^{\prime}} d y_{1} \wedge d y_{2} \wedge d y_{3}=G \sqrt{\operatorname{det} g} d y_{1} \wedge d y_{2} \wedge d y_{3}=*^{\prime} \mathbf{1}
$$

Here $y_{i}$ are the coordinates of the 3 -torus and $*^{\prime}$ is the Hodge star operator of the deformed geometry. The form of $G=\operatorname{det}(C E+D)^{-1}$ for a general 3-parameter deformation, for which $D=I$ and $C=\Gamma$ in (4.1), was given in (4.14). First thing we observe is that $G$ has no zeroes, so starting with a non-singular solution, we end up with a non-singular solution, unless $G$ blows up at some points. In general, as $\Delta_{i}$ and $K_{i}$ can become negative, $G$ may have poles for some backgrounds, when $\gamma_{i}$ are related. Let us make a case by case analysis for the examples considered here. In the case of $A d S_{5} \times S^{5}, K_{i}=0$ and $\Delta_{i} \geq 0$, so $G$ does not have any poles in either of the one-parameter and three-parameter deformations. For one-parameter deformations of $T^{1,1}$ and $Y^{p, q}$, for which $\gamma_{1}=\gamma_{2}=0$, there is no contribution from $K_{i}$, and $\Delta_{3} \geq 0$, so again, $G$ has no poles. Now consider the new three-parameter deformation of $T^{1,1}$, which we gave in (4.11). Here too, $G$ has no poles, as can be seen easily when written in the following form:

$$
G^{-1}=\frac{1}{108}\left(108+2 s_{\theta_{1}}^{2}\left(c_{\theta_{2}} \gamma_{3}-\gamma_{2}\right)^{2}+2 s_{\theta_{2}}^{2}\left(c_{\theta_{1}} \gamma_{3}-\gamma_{1}\right)^{2}+3 \gamma_{3}^{2} s_{\theta_{1}}^{2} s_{\theta_{2}}^{2}\right) .
$$

For the analysis of $Y^{p, q}$, one should first notice that the range of $y$ is fixed such that $1-y>0, a-y^{2}>0, w(y)>0, q(y) \geq 0$ [24]. From this, one immediately sees that $\Delta_{i} \geq 0$. However, $K_{1}$ and $K_{2}$ can become negative at some points. For example, $K_{1}<0$ when $\pi / 2<\theta<\pi$. So, for some points or on some lines or planes in the parameter space, $G$ might have poles at some values of $\theta$ and $y$. Note that the plane $\gamma_{3}=0$ is safe in the parameter space, as then there is no contribution from $K_{1}$ or $K_{2}$.

The next thing to be noticed about the new three-parameter deformations found here is that it does not reduce to the undeformed solution when the volume form of the threetorus goes to zero. As can be seen from (4.13), when $e=0$, the metric scales as $g_{i j} \rightarrow G g_{i j}$ and the B-field does not vanish. However, when the volume forms of all the three 2-tori within the 3-torus are zero, that is, when $\Delta_{1}=\Delta_{2}=\Delta_{3}=0$ (which immediately implies that $K_{1}=K_{2}=K_{3}=e=0$ ), our solution reduces to the undeformed solution.

Finally, an important criteria for the regularity of the new solutions is that the RR field $C_{2}$ should vanish, as the internal torus shrinks to zero size [1]. In the previous section, we have seen that $F_{3}=d C_{2}$ is schematically of the form

$$
F_{3}=\frac{(16 \pi N) \pi^{3}}{\operatorname{Vol}\left(X_{5}\right)} \sqrt{g\left(X_{5}\right)} d y_{1} \wedge d y_{2} \wedge \sum_{i=1,2,3} \gamma_{i} d y_{i}
$$


where $\sqrt{g\left(X_{5}\right)} d y_{1} \wedge d y_{2} \wedge d y_{3} \wedge d y_{4} \wedge d y_{5}$ is the volume form of $X_{5}$ and $d y_{i}, i=1,2,3$ are the coordinates of the 3-torus of isometries, $X_{3}$. So the behavior of $C_{2}$ as the 3-torus shrinks depends entirely on how the volume form of $X_{3}$ is related to that of $X_{5}$. In the case of $T^{1,1}, \sqrt{g\left(X_{5}\right)}=\frac{1}{108} s_{\theta_{1}} s_{\theta_{2}}=\frac{1}{36} \sqrt{g\left(X_{3}\right)}$, so $F_{3}$ vanishes at the zeroes of the volume form of $X_{3}$. At these points $C_{2}$ is pure gauge and as such it can be gauged away. However, in the case of $Y^{p, q}$

$$
\sqrt{g\left(X_{5}\right)}=\frac{l(1-y) s_{\theta}}{18}, \quad \sqrt{g\left(X_{3}\right)}=\frac{l s_{\theta}}{3} \sqrt{\frac{2 y^{3}-3 y^{2}+a}{3}} .
$$

We see that $C_{2}$ does not necessarily vanish as the 3 -torus of isometries shrinks to zero size.

\section{Conclusions}

In this paper, we have examined the solution generating symmetries used by [1] and identified the $O(2,2, \mathbb{R})$ matrix that acts on $E=g+B$. The solution generating $O(2,2, \mathbb{R})$ acts locally on the moduli space of two dimensional conformal field theories on the worldsheet, taking one CFT to another connected to it by an exactly marginal deformation. Correspondingly, the dual field theory on the boundary of $A d S_{5}$ is deformed by an exactly marginal operator, giving rise to $\beta$ deformations of the original theory. On the other hand, the discrete $O(2,2, Z)$ takes the world-sheet CFT to an equivalent one, just like the corresponding deformation of the field theory with integer $\beta$ gives the same theory that one has started with. We have seen that some of the important features of the deformed gravity backgrounds follow from the properties of the $O(2,2, \mathbb{R})$ matrix.

We found here a new 3-parameter family of deformations of the Sasaki-Einstein manifolds $T^{1,1}$ and $Y^{p, q}$. We have seen that the new solutions associated with $T^{1,1}$ are regular, whereas there are some problems with the regularity of the deformations of $Y^{p, q}$.

An obvious next step would be to find the Lax pair of these new backgrounds following the methods developed by [2]. It would also be interesting to examine the associated spin chain as was done in [4] for the $A d S_{5} \times S^{5}$ case.

\section{Acknowledgements}

I am grateful to Sergey Cherkis for helpful discussions, for reading the manuscript and for useful suggestions. I also would like to thank Chris Hull and Dan Waldram for helpful e-mail correspondence. This work is supported by Irish Research Council for Science, Engineering and Technology (IRCSET) under the postdoctoral fellowship scheme. 


\section{References}

[1] O. Lunin and J. Maldacena, "Deforming field theories with U(1) x U(1) global symmetry and their gravity duals," JHEP 0505 (2005) 033 [arXiv:hep-th/0502086].

[2] S. Frolov, "Lax pair for strings in Lunin-Maldacena background," JHEP 0505, 069 (2005) [arXiv:hep-th/0503201].

[3] S. A. Frolov, R. Roiban and A. A. Tseytlin, "Gauge - string duality for superconformal deformations of $\mathrm{N}=4$ super Yang-Mills theory," JHEP 0507, 045 (2005) [arXiv:hep-th/0503192].

[4] N. Beisert and R. Roiban, "Beauty and the twist: The Bethe ansatz for twisted N $=4$ SYM,” JHEP 0508, 039 (2005) [arXiv:hep-th/0505187].

[5] S. A. Frolov, R. Roiban and A. A. Tseytlin, "Gauge-string duality for (non)supersymmetric deformations of $\mathrm{N}=4$ super Yang-Mills theory," Nucl. Phys. B 731, 1 (2005) [arXiv:hep-th/0507021].

[6] R. G. Leigh and M. J. Strassler, "Exactly marginal operators and duality in four-dimensional N=1 supersymmetric gauge theory," Nucl. Phys. B 447, 95 (1995) [arXiv:hep-th/9503121].

[7] O. Aharony, S. S. Gubser, J. M. Maldacena, H. Ooguri and Y. Oz, "Large N field theories, string theory and gravity," Phys. Rept. 323 (2000) 183 [arXiv:hep-th/9905111].

[8] D. Berenstein and S. A. Cherkis, "Deformations of N =4 SYM and integrable spin chain models," Nucl. Phys. B 702, 49 (2004) [arXiv:hep-th/0405215].

[9] U. Gursoy and C. Nunez, "Dipole deformations of N = 1 SYM and supergravity backgrounds with U(1) x U(1) global symmetry," Nucl. Phys. B 725, 45 (2005) [arXiv:hep-th/0505100].

[10] C. h. Ahn and J. F. Vazquez-Poritz, "Marginal deformations with U(1)**3 global symmetry," JHEP 0507 (2005) 032 [arXiv:hep-th/0505168]; C. h. Ahn and J. F. Vazquez-Poritz, "Deformations of flows from type IIB supergravity," [arXiv:hep-th/0508075].

[11] J. P. Gauntlett, S. Lee, T. Mateos and D. Waldram, "Marginal deformations of field theories with AdS(4) duals," JHEP 0508, 030 (2005) [arXiv:hep-th/0505207].

[12] R. de Mello Koch, J. Murugan, J. Smolic and M. Smolic, "Deformed PP-waves from the Lunin-Maldacena background," JHEP 0508 (2005) 072 [arXiv:hep-th/0505227]; R. de Mello Koch, N. Ives, J. Smolic and M. Smolic, "Unstable giants," [arXiv:hep-th/0509007].

[13] T. Mateos, "Marginal deformation of N =4 SYM and Penrose limits with continuum spectrum," JHEP 0508 (2005) 026 [arXiv:hep-th/0505243].

[14] D. Z. Freedman and U. Gursoy, "Comments on the beta-deformed N = 4 SYM theory," JHEP 0511 (2005) 042 [arXiv:hep-th/0506128].

[15] S. Penati, A. Santambrogio and D. Zanon, "Two-point correlators in the beta-deformed N = 4 SYM at the next-to-leading order," JHEP 0510 (2005) 023 [arXiv:hep-th/0506150], A. Mauri, S. Penati, A. Santambrogio and D. Zanon, "Exact results in planar N = 1 superconformal Yang-Mills theory," JHEP 0511 (2005) 024 [arXiv:hep-th/0507282].

[16] H. Y. Chen and S. Prem Kumar, "Precision test of AdS/CFT in Lunin-Maldacena background," [arXiv:hep-th/0511164].

[17] L. F. Alday, G. Arutyunov and S. Frolov, "Green-Schwarz Strings in TsT-transformed backgrounds," [arXiv:hep-th/0512253]. 
[18] I. R. Klebanov and E. Witten, "Superconformal field theory on threebranes at a Calabi-Yau singularity," Nucl. Phys. B 536, 199 (1998) [arXiv:hep-th/9807080].

[19] D. Martelli and J. Sparks, "Toric geometry, Sasaki-Einstein manifolds and a new infinite class of AdS/CFT duals," [arXiv:hep-th/0411238].

[20] S. Benvenuti, S. Franco, A. Hanany, D. Martelli and J. Sparks, "An infinite family of superconformal quiver gauge theories with Sasaki-Einstein duals," JHEP 0506, 064 (2005) [arXiv:hep-th/0411264].

[21] C. M. Hull and P. K. Townsend, "Unity of superstring dualities," Nucl. Phys. B 438, 109 (1995) [arXiv:hep-th/9410167].

[22] A. Giveon, M. Porrati and E. Rabinovici, "Target space duality in string theory," Phys. Rept. 244, 77 (1994) [arXiv:hep-th/9401139].

[23] A. Giveon and M. Rocek, "Generalized duality in curved string backgrounds," Nucl. Phys. B 380, 128 (1992) [arXiv:hep-th/9112070].

[24] J. P. Gauntlett, D. Martelli, J. Sparks and D. Waldram, "Sasaki-Einstein metrics on S(2) x S(3)," Adv. Theor. Math. Phys. 8 (2004) 711 [arXiv:hep-th/0403002]; J. P. Gauntlett, D. Martelli, J. F. Sparks and D. Waldram, "A new infinite class of Sasaki-Einstein manifolds," [arXiv:hep-th/0403038].

[25] J. G. Russo, "String spectrum of curved string backgrounds obtained by T-duality and shifts of polar angles," JHEP 0509, 031 (2005) [arXiv:hep-th/0508125].

[26] M. J. Duff, H. Lu and C. N. Pope, "Supersymmetry without supersymmetry," Phys. Lett. B 409, 136 (1997) [arXiv:hep-th/9704186], M. J. Duff, H. Lu and C. N. Pope, "AdS(5) x S(5) untwisted," Nucl. Phys. B 532, 181 (1998) [arXiv:hep-th/9803061].

[27] C. Hull, "Holonomy and symmetry in M-theory," [arXiv:hep-th/0305039].

[28] E. Bergshoeff, C. M. Hull and T. Ortin, "Duality in the type II superstring effective action," Nucl. Phys. B 451, 547 (1995) [arXiv:hep-th/9504081].

[29] M. Cvetic, H. Lu, C. N. Pope and K. S. Stelle, "T-duality in the Green-Schwarz formalism, and the massless/massive IIA duality map," Nucl. Phys. B 573, 149 (2000) [arXiv:hep-th/9907202].

[30] S. F. Hassan, "T-duality, space-time spinors and R-R fields in curved backgrounds," Nucl. Phys. B 568, 145 (2000) [arXiv:hep-th/9907152].

[31] M. Fukuma, T. Oota and H. Tanaka, "Comments on T-dualities of Ramond-Ramond potentials on tori," Prog. Theor. Phys. 103, 425 (2000) [arXiv:hep-th/9907132].

[32] E. Witten, "String theory dynamics in various dimensions," Nucl. Phys. B 443, 85 (1995) [arXiv:hep-th/9503124].

[33] E. Cremmer, B. Julia, H. Lu and C. N. Pope, "Dualisation of dualities. II: Twisted self-duality of doubled fields and superdualities," Nucl. Phys. B 535, 242 (1998) [arXiv:hep-th/9806106]. 